Western University

Scholarship@Western

Business Publications

Business (Richard Ivey School of Business)

Fall 10-29-2017

\title{
Contracts to Promote Optimal Use of Optional Diagnostic Tests in Cancer Treatment
}

Salar Ghamat

Ivey Business School, sghamat.phd@ivey.ca

Gregory S. Zaric

University of Western Ontario, gzaric@ivey.ca

Hubert Pun

IveyBusiness School, hpun@ivey.uwo.ca

Follow this and additional works at: http://ir.lib.uwo.ca/iveypub

Part of the Management Sciences and Quantitative Methods Commons, and the Operations and Supply Chain Management Commons

Citation of this paper:

Ghamat, Salar; Zaric, Gregory S.; and Pun, Hubert, "Contracts to Promote Optimal Use of Optional Diagnostic Tests in Cancer Treatment" (2017). Business Publications. 2.

http://ir.lib.uwo.ca/iveypub/2 


\title{
Contracts to Promote Optimal Use of Optional Diagnostic Tests in Cancer Treatment
}

\author{
Salar Ghamat (Corresponding author) \\ Lazaridis School of Business and Economics, Wilfrid Laurier University \\ 75 University Ave W, Waterloo, Ontario, Canada \\ (519) 884-0710 x2439 \\ sghamat@wlu.ca
}

Gregory S. Zaric

Ivey Business School, Western University

1255 Western Road, London, Ontario, Canada

(519) 661-3415

gzaric@ivey.uwo.ca

Hubert Pun

Ivey Business School, Western University

1255 Western Road, London, Ontario, Canada

(519) 850-2452

hpun@ivey.uwo.ca

"This is the peer reviewed version of the following article: Ghamat, S. et al. Contracts to Promote Optimal Use of Optional Diagnostic Tests in Cancer Treatment. Production and Operations Management (2017), which has been published in final form at https://doi.org/10.1111/poms.12780. This article may be used for non-commercial purposes in accordance with Wiley Terms and Conditions for Self-Archiving." 


\title{
Contracts to Promote Optimal Use of Optional Diagnostic Tests in Cancer Treatment
}

\begin{abstract}
In this paper we examine performance-based payment contracts to promote the optimal use of an optional diagnostic test for newly diagnosed cancer patients. Our work is inspired by three trends: tremendous increases in the cost of new, advanced cancer drugs; development of new diagnostic tests to allow physicians to tailor treatment to patients; and changes in healthcare funding models that reward quality care. We model the interaction between two parties - a healthcare payer and an oncologist, in which the oncologist has private information about patients' characteristics (adverse selection) and the payer does not know whether the oncologist takes the optimal course of action (moral hazard). We show that, in the presence of information asymmetry, a healthcare payer should never incentivize an oncologist to use a diagnostic test for all patients, even if the diagnostic test is available for free. Moreover, although the oncologist has additional information about a patient's risk, he cannot always benefit from this private information. We also find that social welfare may not increase as a result of a decrease in the oncologist's concerns regarding the health outcome of patients. Finally, we show that it is not always socially optimal to make a diagnostic test compulsory even if such a policy can be implemented for free.
\end{abstract}

Keywords: contract design; game theory; performance-based contracts; personalized medicine; quality of care

Received: February 2016; accepted: August 2017 by Sergei Savin after three revisions.

\section{Introduction}

Cancer is the second leading cause of death in the United States, accounting for over 580,000 deaths in 2013 (Center for Disease Control and Prevention 2013). The American Cancer Society estimates that there will be over 1,680,000 new cancer diagnoses in 2016 (American Cancer Society 2016). Cancer treatment typically consists of a combination of surgery, radiation therapy, hormone treatment, and chemotherapy. In recent years, cancer treatment costs have risen dramatically with the development of a number of biologics and targeted treatments. For 
example, the cost of a course of therapy with trastuzumab (Herceptin) for breast cancer is estimated at $\$ 30,000$ (Hornberger et al. 2005); the cost of a course of therapy with bevacizumab (Avastin) for colorectal cancer is approximately $\$ 90,000$ (Picard 2012), and other drugs have been developed with a wide range of monthly costs per patient (Campbell 2015).

In addition to growth in the number and cost of treatment options available, there has also been growth in the availability and use of personalized medicine, often implemented through genetic and gene-expression profiling tests, which can help guide cancer diagnosis and treatment decisions. In some jurisdictions, a KRAS test is required prior to treatment with cetuximab or panitimuab for colorectal cancer, based on evidence that these drugs are not effective in patients with mutations in the KRAS gene (Díaz-Rubio et al. 2012). The 21-gene assay for breast cancer (Oncotype Dx) generates a recurrence score based on the expression of 21 genes. The score is interpreted as the probability of cancer recurrence within 10 years and is used to guide adjuvant chemotherapy treatment decisions. In particular, women with a high recurrence score are advised to undergo adjuvant chemotherapy, whereas women with a low recurrence score may avoid adjuvant chemotherapy as it provides limited benefits but is associated with harmful side effects. A gene expression profiling test for cancer of unknown primary (the Tissue of Origin test) examines the expression of more than 2,000 genes and produces a set of 15 "similarity scores" that are interpreted as the probability that the tumor is one of 15 common types. This test can help to guide treatment decisions by directing treatment towards the most appropriate agents for a given type of cancer instead of using a general, non-specific treatment regimen. These diagnostic tests are an important element of personalized medicine since individualized treatment cannot be prescribed in the absence of this type of information. 
The costs of these tests vary from less than $\$ 500$ for single-gene tests like KRAS (Behl et al. 2012) to $\$ 4,000$ or more for multi-gene tests (e.g., Oncotype Dx costs $\$ 4,175$ (Ray 2011) and Tissue of Origin costs $\$ 3,750$ (Tansey 2008)). Use of many of these tests is supported by clinical guidelines (Allegra et al. 2009, Carlson and Roth 2013) and a number of health technology assessments (e.g., Hannouf et al. 2012, Nerurkar et al. 2014, Hannouf et al. 2016). However, the uptake of these advanced tests is low despite evidence of benefits. For example, Segurado (2016) writes "despite solid scientific evidence and endorsement by oncology societies for multi-gene tests supporting therapy decision-making in breast cancer, only a small percentage of women are able to safely skip chemotherapy through personalized medicine." In the United States, 90 percent of people have insurance plans that cover the Oncotype Dx test, yet only 27 percent of eligible cancer patients used the test in 2010 (Enewold 2015).

To help manage chemotherapy costs and incentivize physicians to provide high-quality care, the Centers for Medicare and Medicaid Services (CMS) has developed a new funding model called the Oncology Care Model (OCM). The OCM payment scheme consists of a "target price" for a six-month episode of treatment that is based on the average treatment cost of patients with similar health conditions. Under OCM, providers receive payments based on a fee-forservice (FFS) model where the providers are compensated based on the volume of services performed. At the end of each treatment episode the providers may receive reward payments, conditional on the patient's health status, if the total cost of treatment is below the target price (CMS 2015). The OCM thus creates conflicting objectives in managing care: while the target price aims to limit the treatment cost, the reward payments give physicians the flexibility of choosing more expensive treatments if it is believed that they will have a positive impact on the patient's health status. 
The OCM is part of a more general type of payment reform that focuses on performancebased bundled payments. For instance, CMS introduced a 5-year payment program in April 2016 for lower extremity joint replacement called the Comprehensive Care for Joint Replacement (CJR) Model. Under the CJR model, hospitals will be compensated based on a target price that aims to cover the total cost of an episode of treatment. Hospitals may also receive quality incentive payments based on the health outcome of patients. Recent studies suggest that there is growing interest in bundled payment models by healthcare payers (Japsen 2015, Whitman 2016). Bundled payment models accounted for 15 percent of insurers' spending in 2014 (Evans 2014) and there is evidence that these payment models have resulted in positive outcomes (Korda and Eldridge 2011).

In this paper, we develop a stylized physician compensation model inspired by the growing use of advanced diagnostic testing technologies in cancer care. Although diagnostic tests are not explicitly included in OCM, the growing number of expensive testing technologies and the need to ensure the appropriate use of these tests suggests a need to include them in future funding models. Thus, our research explores what a bundled payment mechanism should look like if diagnostic tests are available. The central problem analyzed in this paper was identified in a report by consulting firm McKinsey and Co., which described one of the barriers to adopting new testing technologies for personalized medicine as "the difficulty of enforcing standard protocols to ensure that physicians follow through with appropriate patient care based on test results" (Davis et al. 2010).

Our model involves interaction between a healthcare payer (payer) who pays for the test and advanced treatment (e.g., adjuvant chemotherapy), and an oncologist (provider) who makes decisions regarding the use of the test and the advanced treatment for patients. Patients are 
heterogeneous, with different risks of cancer recurrence. Since the recurrence probability can only be known if the test is used, patients appear to be homogeneous members of different risk groups if the test is not used. The payer offers a payment contract to providers to maximize social welfare, defined as the monetary value of health outcomes minus the total costs of providing care. Depending on the payment structure offered by the payer, the provider chooses one of three treatment options: 1) Treat all patients with an advanced treatment without ordering the test; 2) Do not order the test and do not treat with an advanced treatment; or 3) Order the test and only treat those patients with advanced treatment whose recurrence probability is sufficiently high. While in the main analysis we assume that the provider decides whether to use the test or not, in some cases the test can be made compulsory (e.g., KRAS); therefore, in Section 6 we extend our analysis to the case where the test is compulsory for all patients.

The contract design problem is complicated by information asymmetry in the system. First, there are many patient characteristics that are observed by physicians but are not formally recorded in any clinical or administrative databases held by the payer (adverse selection). Second, the payer also does not know which factors the physician considered when making the treatment decision, so the payer does not know whether the optimal course of action is used by the provider (moral hazard). To incentivize the provider to use the test whenever it is beneficial and to appropriately follow the recommendation of the test results whenever the test is used, the payer offers a menu of contracts consisting of a fixed payment per episode of treatment, and reward payments that are based on patients' health and on whether or not advanced treatment is applied.

The underlying problem has a structural novelty in that the provider's decision to use an advanced treatment is conditional on his decision to order the diagnostic test. Therefore, finding 
the optimal menu of contracts is a challenging problem as the constraints in the payer's problem are not continuous. However, we construct the optimal menu of contracts by solving the payer's problem for each treatment choice of the provider.

Our paper yields several interesting findings that have policy implications. First, we show how the payer can design contracts such that the provider may not be able to take advantage of his private information. In this optimal contract structure, the payer should incorporate the provider's treatment choices in the contracts. In particular, the payer should offer reward payments for not treating certain types of patients with an advanced treatment. This finding underlines empirical evidence on over-prescription of chemotherapy treatment (Smith and Hillner 2010) and suggests that "quality care" does not always depend on using the most expensive technology available. Furthermore, it shows that the advent of new diagnostic tests necessitates new reimbursement models because current payment models do not account for treatment choices made by the providers.

Second, if the test is relatively inexpensive, then it may seem natural that the provider should order the diagnostic test for all patients. Interestingly, when the test is optional and there is imperfect information flow between the payer and the provider, we find that the payer should motivate the provider to only test certain types of patients, even if the diagnostic test can be available for free. This is because avoiding patient misrepresentation, when testing all patients, requires higher-valued contract payment terms compared to the case where the test is used for only one patient type. This is in agreement with empirical evidence showing that some new technologies might not be cost-effective even if they can be acquired for free (Davis 2014, Davis and Akehurst 2016). However, under complete information it is optimal to test all patients when the test cost is sufficiently low. This result highlights the importance of information flow in the 
healthcare system and provides the first step in estimating the value gained by enhancing administrative databases held by the payer.

Third, we find that a decrease in the reputational concerns and altruistic behaviour of a provider may not increase social welfare. This result is in line with the inconsistent evidence regarding the impact of malpractice pressure on healthcare systems (Avraham and Schanzenbach 2010, Reyes 2010, Cotet 2012, Lakdawalla and Seabury 2012, Dai et al. 2016, Montanera 2016). Thus, we provide further support for Montanera (2016) who argues that tort reform, even if successful at reducing malpractice pressure, is not a "silver bullet" capable of achieving both cost reductions and quality improvements for all patients.

Finally, we show that it is not always socially optimal to make a diagnostic test compulsory even if there is no cost to implement such a policy. While compulsory testing could eliminate adverse selection, it causes unnecessary testing of patients who are very likely to benefit (or not benefit) from an advanced treatment based on their pre-test evaluation. Thus, this finding suggests that even if a diagnostic test is recommended by clinical guidelines, it might be better to leave the decision about ordering the test to the judgment of physicians instead of making the test compulsory for the whole population.

\section{Literature Review}

Our study is primarily related to the stream of literature that considers design of mechanisms (e.g., performance-based payment models) for healthcare systems. For example, there are studies that design performance-based contracts to improve patient access to care (e.g., Jiang et al. 2012, Jiang et al. 2016, Savva et al. 2016). Jiang et al. (2012) consider contracts between a payer and a healthcare provider that serves a heterogeneous patient pool using an online appointmentscheduling system. They propose a threshold penalty-payment scheme to reach a desired patient 
waiting time (performance measure). Jiang et al. (2016) and Savva et al. (2016) study performance-based incentives when there is direct competition among providers. In these studies, performance measure is included in the proposed contracts. However, in our study, performance measure that is a patient's health outcome is probabilistic and cannot be included as a contract term. Fuloria and Zenios (2001) also design performance-based contracts motivated by Medicare's End Stage Renal Disease (ESRD) program, which was established in 1973 to provide affordable dialysis care for ESRD patients. They assume that all patients are homogeneous with the same probability function of experiencing complications or death after treatment. Our work differs in that we study heterogeneous patients that can only be sorted using a diagnostic test. Finally, Gupta and Mehrotra (2015) study the proposer selection problem of CMS for its "bundled payments for care improvement" initiative. Similar to our study, they find coordinating mechanisms to align the incentives of the payer and the provider. However, we focus on the heterogeneous patient pool visiting a provider that is already a participant of a payment program, while they focus on the provider competition and payer's strategy to get optimal proposals from the providers.

There are also studies in healthcare operations management that analyze a provider's choice regarding use of diagnostic tests in various healthcare settings. Motivated by the utilization of cancer screening tests, Yaesoubi and Roberts (2011) study optimal reimbursement contracts between a payer and a healthcare provider. The provider is assumed to only care about the cost of treatment, and thus the health outcome of a patient does not affect the treatment choice of the provider. Conversely, we consider the case where the provider is concerned about patients' health outcome. Moreover, we study the effects of an optional diagnostic test that can help the provider when deciding on treatment, whereas in Yaesoubi and Roberts (2011), the use 
of a particular test is the provider's only decision. Dai et al. (2016) study the effect of different service environments (e.g., provider's misdiagnosis concern, reimbursement ceiling) on the provider's test-ordering behaviour. Their study is focused on general diagnostic tests, such as MRI or EKG, that are used to identify whether a patient has a disease. In contrast, we are interested in personalized medicine and diagnostic tests that identify if a patient (who is already diagnosed with a disease) can benefit from a targeted therapy. Similar to our approach to modeling the provider's altruistic behaviour and reputational concerns when patients are not properly treated, Dai et al. (2016) explicitly modeled the provider's misdiagnosis concern and show that both over-testing and under-testing are possible outcomes of these concerns. Finally, Dai et al. (2015) study the use of an optional test on decision-making of a provider regarding the use of percutaneous coronary intervention (PCI) for coronary artery disease. Similar to our approach they assume some degree of altruistic behaviour by providers and model it as a conflict of interest between financial gains and patients' health outcome. They show that the incorporation of optional advanced testing into decision-making might have non-monotonic effects on the use of PCI depending on the amount of the provider's conflict of interest. Unlike our study, they do not incorporate the payer's incentives in use of the optional test and do not study the potential payment systems that might align the incentives of the payer and the provider.

Adida et al. (2016) study the effects that FFS and bundled payment models have on patient selection and the treatment intensity decisions made by a provider. Similar to our model they consider a reputational penalty for the provider in case of a treatment failure. They find that the FFS model would result in overtreatment of patients. Guo et al. (2016) also compares the FFS model with a bundled payment model in a setting where patients are sensitive to waiting times. They show that when the patient pool is sufficiently small the bundled payment model 
dominates the FFS model in all performance measures. Similar to our study they find optimal reimbursement policy of a payer who maximizes the patient welfare. Andritsos and Tang (2015) consider a co-managed situation where both the provider and the patient can exert effort to reduce the readmission rate. They show that bundled payment outperforms FFS by causing the provider and the patient to exert more effort to reduce readmission. These studies investigate incentive problems in healthcare systems in order to compare the outcome of different payment models, but they do not consider performance-based bundled payments. In this paper, we model newly proposed payment models that reward providers for higher quality of care and design performance-based payment contracts that align the incentives of the payer and the provider while achieving socially optimal treatment levels. To the best of our knowledge, we are the first to study the use of performance-based contracts to coordinate the incentives of a healthcare payer and a provider while examining the impact of an optional diagnostic test on the provider's decision-making process.

\section{The Model}

We consider a system that consists of a payer, a provider, and a pool of newly diagnosed patients who are eligible for an optional diagnostic test which shows the potential health benefits that a patient may receive from an advanced treatment option. We extend our analysis to a case where the diagnostic test is compulsory in Section 6. In this section, we consider a system with adverse selection (the provider has private information about the patients' risk profile) and moral hazard (the payer does not know whether or not the treatment that is recommended by the test result is followed). The provider makes the testing and treatment decisions, and the payer incentivizes the provider with a payment contract in order to maximize social welfare. Consistent with current practice for the Oncotype Dx and Tissue of Origin tests, we assume that the test result is sent 
directly to the provider. Throughout the paper we use the term "advanced treatment" to mean the provider is treating the patient with a specific treatment (e.g., chemotherapy or a targeted treatment), that would be recommended from the results of a diagnostic test. We use the term "basic treatment" for a base case scenario where the provider is not treating the patient with an advance treatment, but instead treat the patient by other means that are appropriate given her pretest diagnosis (e.g., hormone therapy or empiric chemotherapy). All costs and benefits of the advanced treatment are assumed to be relative to the costs and benefits of the basic treatment (that are normalized to zero). The provider incurs a treatment cost for using advanced treatment (e.g., chemotherapy cost plus costs associated with administering treatment and monitoring patients) and is compensated via his contract with the payer, while the test cost is directly billed to the payer.

The provider's patients can have different risk levels. The risk profile of the patients is private information to the provider because the provider is able to make several observations about each patient that are not known to the payer. An example of private information is "Has a stable lifestyle", which can influence many factors such as adherence to medication recommendations and preventive measures to prevent recurrence, and thus influence the success of a given treatment recommendation. For example, Bangsberg et al. (2000) found a strong inverse relationship between HIV viral load and adherence to protease inhibitors, and Kaposy et al. (2017) described the efforts made in several HIV clinics to monitor and improve adherence among patients with poor adherence. Other examples include detailed family history or various risk factors that are disclosed to a physician but not formally recorded in patient charts. Ferris et al. (2017) recommend incorporating patients' goals and preferences into treatment decisions, but neither of these factors are routinely collected on patient charts. Guidelines from the National 
Cholesterol Education Program recognize the importance of diet and exercise as important modifiable risk factors for coronary heart disease (Grundy et al. 2002), yet this information may not be recorded in insurer or administrative databases in an standardized way, if it is recorded at all. In addition, the results of some lab tests will be sent directly to the physician. Depending on the structure of the payer's administrative databases, the payer may know that a test has been ordered (because of an associated billing code), but not know the result of the test. A proliferation of new tests, each with their own reporting standards, will likely exacerbate this problem. For instance, the Tissue of Origin test reports results as a set of 15 similarity scores, along with a "most likely tissue of origin" and a "degree of confidence". Recording the full results of this test would require customization of existing databases, which may not be a priority for the payer, particularly if the tests are used for rare conditions. The extent of this source of information asymmetry will vary among payers depending on the structure of their health records systems and their sophistication in using the information collected-but even for a sophisticated payer, the physician will know more about a patient than the payer does.

For this analysis, without loss of generality, we normalize the number of patients of the provider to one. We assume that patients can be one of two types: high-risk (type- $H$ ) and lowrisk (type- $L$ ). The recurrence probability (risk score) of type- $i \in\{H, L\}$ patients is characterized by a random variable, $r_{i} \in[0,1]$, with corresponding distributions $F_{H}(r)$ and $F_{L}(r)$ with density functions $f_{H}(r)$ and $f_{L}(r)$. Thus, the expected recurrence probability of type- $i$ patients is $E\left[r_{i}\right]=$ $\int_{0}^{1} y f_{i}(y) d y$. We assume that patients' risk distribution function $F_{i}(r)$ is continuous and strictly increasing on $[0,1]$. Moreover, we assume that $F_{H}(r) \leq F_{L}(r)$ for all $r$, such that type- $H$ patients have a higher expected recurrence probability than type- $L$ patients. The provider can observe the type but does not know a patient's recurrence probability $r_{i}$ unless the patient undergoes the test, 
and ordering the test reveals this patient's recurrence probability to the provider. The payer only knows the probability of the patient type to be of type- $i$ with probability $\beta_{i}$, where $\beta_{L}+\beta_{H}=1$.

We assume that advanced treatment can reduce the recurrence probability to $k r_{i}$, where $0<k<1$ is the advanced treatment's hazard ratio such that the advanced treatment is more effective when the hazard ratio $k$ is small. This assumption is consistent with the use of the 21gene assay for breast cancer where the original validation study found that the difference in the probability of recurrence for women treated with chemotherapy versus hormone therapy alone was increasing in the recurrence score (Paik et al. 2006).

The payer offers a menu of contracts $\zeta_{i}=\left\{w_{i}, b_{t i}, b_{n i}\right\}$ that depends on the risk distribution of patient types (time $t_{0}$; Figure 1). The contracts consist of the following three terms: 1) A fixed bundled payment for an episode of advanced treatment $\left.\left(w_{i}\right), 2\right)$ a reward for stable health of patients treated with advanced treatment $\left(b_{t i}\right)$, and 3) a reward for stable health of patients treated with basic treatment $\left(b_{n i}\right)$. The first term of the contract $\left(w_{i}\right)$ only pays for quantity of treatment and captures the most widely implemented payment method in different healthcare systems. For example, $w_{i}$ can be interpreted as FFS payment when it covers the cost of an episode of advanced treatment. However, when $w_{i}$ is different from the total cost of the advanced treatment it can be interpreted as a target price (cf. CJR model) - meaning that the provider might receive more or less than the total cost of the advanced treatment. The next two terms are the performance-based payments that depend on the health outcome of patients as well as the treatment choice of the provider. After the payment contracts are offered by the payer, a patient will arrive $\left(t_{1}\right)$. The patient type is either low-risk or high-risk, which is observable to the provider but not to the payer. Next, the provider will decide whether or not to order the 
diagnostic test $\left(t_{2}\right)$. If the test is ordered the provider will receive the test results from the lab $\left(t_{3}\right)$ and decide whether to use the advanced treatment given the risk score of the patient. However, if the test is not ordered the provider will make the decision on using the advance treatment only based on the patient's type. The provider will treat the patient either using the advanced or the basic treatment $\left(t_{4}\right)$. Finally, the provider will receive the episode payment after the treatment $\left(t_{5}\right)$ and the reward payments after the health outcome of the patient is realized $\left(t_{6}\right)$. Figure 1 illustrates the sequence of events.

Figure 1: Sequence of events

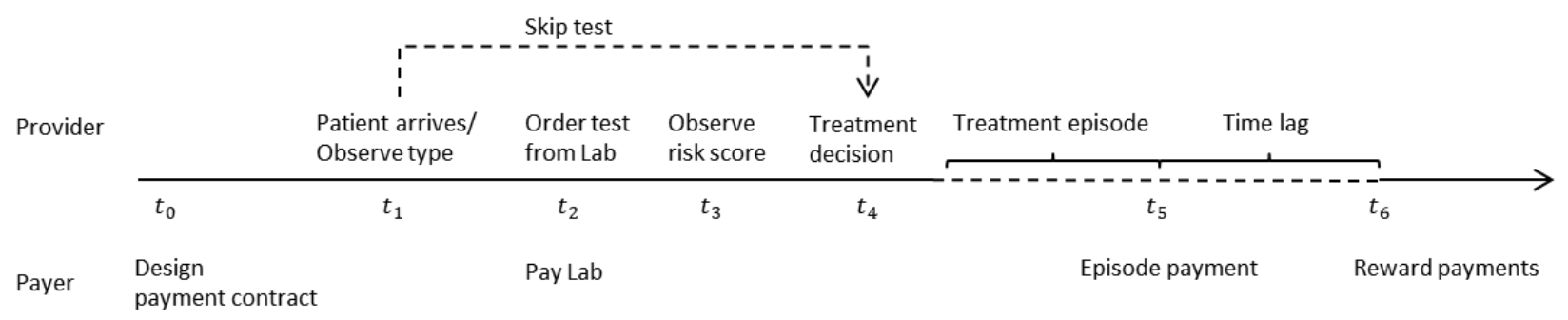

An episode of advanced treatment costs $c$ for each patient. Every patient with recurrence who only received basic treatment incurs a welfare loss $h$ that represents both the opportunity cost and psychological cost of a patient who is not properly treated. Thus, a payer that maximizes social welfare has to account for this welfare loss. We assume that this welfare loss will also cause a disutility $\alpha h$ for the provider. Existing literature has explained the provider's disutility as being either a result of a loss of reputation (Adida et al. 2016) or disutility caused by altruism about patients' health (Montanera 2016). We do not argue for either but instead claim $\alpha h$ to be an aggregate value representing both effects. When $\alpha=0$, adverse patient outcomes cause no disutility for the provider - i.e., no damage to the provider's reputation, and no harm associated with the provider's concerns for the welfare of his patients. We examine the impact of $\alpha$ on social welfare in Section 5. 
There is a net monetary benefit $\lambda_{s}$ associated with a patient with no recurrence (i.e., stable health) and $\lambda_{r}$ in case of recurrence, $\lambda_{r}<\lambda_{s}$. The advanced treatment may be associated with toxicities and adverse events. These cause the payer's net monetary benefit to decrease by $z$, which is the expected cost of adverse events. We assume that $z$ is independent of $r$ on the grounds that adverse events are driven by specific drug regimens, whereas $r$ is driven by genetic risk factors. This is a common assumption in models used to evaluate cost-effectiveness of 21gene assay (e.g., Hornberger et al. 2005, Hornberger et al. 2011, Hannouf et al. 2012, Vanderlaan et al. 2011).

\subsection{Provider's problem}

For each patient, the provider has to decide whether or not to order the test and whether or not to use the advanced treatment option.

Remark 1: If the provider orders the test, then the provider chooses a threshold $\tau_{i}$ for each patient type and only uses advanced treatment for patients whose recurrence probability is higher than this threshold (i.e., $r_{i}>\tau_{i}$ ). This is a result of the recurrence probability being continuous and monotonic, and the treatment effect being proportional to the recurrence probability (i.e., $\left.k r_{i}\right)$.

Because the risk score of a patient is only revealed if the test is ordered, the treatment threshold $\left(\tau_{i}\right)$ decision of the provider is conditional on his test-ordering decision. We use a binary variable $x_{i} \in\{0,1\}$ to denote whether the provider orders the test $\left(x_{i}=1\right)$ or not $\left(x_{i}=0\right)$ when patients are of type- $i$. Thus, for a given patient type, the provider has three options (Figure 2): Option 0 - treat patients with advanced treatment without ordering the test; Option 1 - do not order the test and treat patients with basic treatment; and Option 2 - order the test and use 
advanced treatment only if a patient's recurrence probability is higher than a treatment threshold $\left(r_{i}>\tau_{i}\right)$

Figure 2: Provider's treatment choices

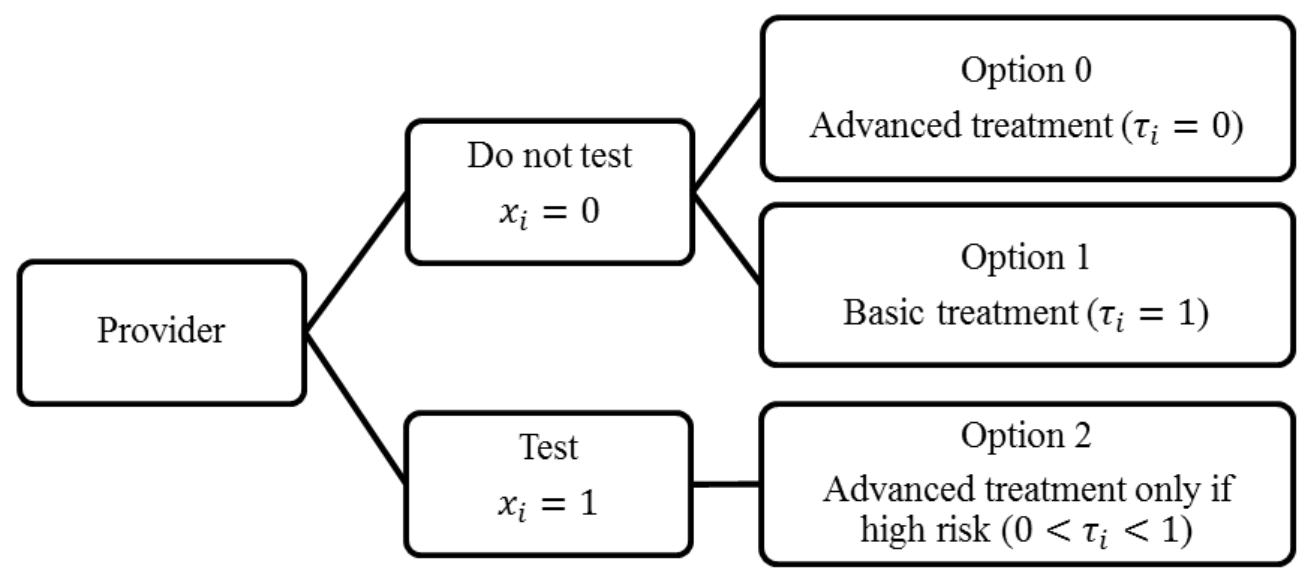

If the provider chooses to treat patients with advanced treatment without ordering the test (Option 0$)$, then the provider's objective function $g_{i}\left(x_{i}, \tau_{i}\right)$ is:

$g_{i}(0,0)=w_{i}-c+b_{t i} \int_{0}^{1}(1-k y) f_{i}(y) d y$

The first two terms are the fixed bundled payment less the cost for an episode of advanced treatment and the third term is the reward payment for patients with stable health who were treated with advanced treatment. If the provider chooses not to order the test and treat patients using basic treatment (Option 1), then the provider's objective function is:

$g_{i}(0,1)=b_{n i} \int_{0}^{1}(1-y) f_{i}(y) d y-\alpha h \int_{0}^{1} y f_{i}(y) d y$

The first term is the reward payment for patients with stable health who were treated with basic treatment. The second term is the provider's disutility when patients who were treated with basic treatment experience a recurrence. Finally, if the provider orders the test (Option 2) and only 
uses advanced treatment if a patient's recurrence score is above the treatment threshold $\left(r_{i}>\tau_{i}\right)$, then the provider's objective function is:

$$
\begin{aligned}
g_{i}\left(1, \tau_{i}\right)=\left(w_{i}-c\right) \int_{\tau_{i}}^{1} f_{i}(y) d y+b_{n i} \int_{0}^{\tau_{i}}(1-y) f_{i}(y) d y+b_{t i} \int_{\tau_{i}}^{1}(1-k y) f_{i}(y) d y \\
\quad-\alpha h \int_{0}^{\tau_{i}} y f_{i}(y) d y
\end{aligned}
$$

The first term is the fixed bundled payment less the cost for an episode of advanced treatment for patients who treated with advanced treatment. The second and the third terms are the reward payments for patients with stable health, and the last term is the provider's disutility when patients who treated with basic treatment experience a recurrence. A similar approach to modeling the disutility associated with adverse outcomes has been widely used in the literature to model the provider's objective function and to incorporate the provider's concerns regarding the health outcome of the patients (e.g., Dai et al. 2015, Adida et al. 2016, Dai et al. 2016, Montanera 2016).

The provider's problem is to maximize his objective function, given the menu of contracts offered by the payer $\zeta_{i}=\left\{w_{i}, b_{t i}, b_{n i}\right\}$, by choosing the treatment choice for each patient type.

\subsection{Payer's problem}

We assume that the payer's objective is to maximize social welfare, which is the monetary value of patients' health outcome minus the total costs of providing care. Thus, assuming the cost of the test is $B$, the payer's objective function, when patients are of type- $i$ is: 


$$
\begin{aligned}
v_{i}\left(\zeta_{i}, x_{i}, \tau_{i}\right)= & \left(\lambda_{s}-b_{n i}\right) \int_{0}^{\tau_{i}}(1-y) f_{i}(y) d y+\left(\lambda_{s}-z-b_{t i}\right) \int_{\tau_{i}}^{1}(1-k y) f_{i}(y) d y \\
& +\left(\lambda_{r}-h\right) \int_{0}^{\tau_{i}} y f_{i}(y) d y+\left(\lambda_{r}-z\right) \int_{\tau_{i}}^{1} k y f_{i}(y) d y-w_{i} \int_{\tau_{i}}^{1} f_{i}(y) d y-x_{i} B
\end{aligned}
$$

The first two terms are the monetary benefit, less the reward payments to the provider, for patients with stable health who are either treated with basic or advanced treatment. The third and the fourth terms are the monetary benefit for patients with recurrence who are either treated with basic or advanced treatment. The fifth and the sixth terms are the payments for an episode of advanced treatment and the test cost. Note that the test cost can only be incurred if the provider orders the test, i.e, setting $x_{i}=1$ under Option 2 . The payer decides on the payment contracts that link the two parties. The payer solves the following program.

$$
\begin{gathered}
\max _{\zeta_{L}, \zeta_{\boldsymbol{H}}} V=\sum_{i \in\{L, H\}} \beta_{i} v_{i}\left(\zeta_{i}, x_{i}, \tau_{i}\right) \\
\text { s.t. }\left\{x_{i}^{*}, \tau_{i}^{*}\right\}=\underset{\substack{x_{i} \in\{0,1\} \\
0 \leq \tau_{i} \leq 1}}{\operatorname{argmax}} g_{i}\left(x_{i}, \tau_{i} \mid \zeta_{i}\right) \\
g_{L}\left(x_{L}^{*}, \tau_{L}^{*} \mid \zeta_{L}\right) \geq \max _{\substack{x_{L} \in\{0,1\} \\
0 \leq \tau_{L} \leq 1}} g_{L}\left(x_{L}, \tau_{L} \mid \zeta_{H}\right) \\
g_{H}\left(x_{H}^{*}, \tau_{H}^{*} \mid \zeta_{H}\right) \geq \max _{\substack{x_{H} \in\{0,1\} \\
0 \leq \tau_{H} \leq 1}} g_{H}\left(x_{H}, \tau_{H} \mid \zeta_{L}\right) \\
g_{L}\left(x_{L}^{*}, \tau_{L}^{*}\right) \geq 0 \\
g_{H}\left(x_{H}^{*}, \tau_{H}^{*}\right) \geq 0
\end{gathered}
$$

The payer's objective function is the total social welfare in a system with information asymmetry. The OTC (optimal treatment choice) constraints are moral hazard incentive constraints to ensure that under the payment contracts $\left\{\zeta_{L}, \zeta_{H}\right\}$ the provider's optimal treatment 
choice is $\left\{x_{i}^{*}, \tau_{i}^{*}\right\}$. The next two constraints (ICL and ICH) are the provider's adverse selection incentive-compatibility constraints. They ensure that the provider prefers contract $\zeta_{i}$ for type- $i$ patients to the contract for the other patient type and thus prevent patient misrepresentation. Note that, if the provider misrepresents a patient he may also choose to change the treatment choice. This is represented by the provider's decision variables in the right-hand side of the ICL and ICH constraints. In particular, $I C L$ constraint means that when a provider faces a low-risk patient, he would be better off choosing the contract that is designed for low-risk patients. Similarly, ICH constraint ensures that a provider would choose a contract designed for high-risk patients when he faces a high-risk patient. The last two constraints (IRL and IRH) are the provider's participation or individual-rationality constraints for each patient type-meaning that the contracts for each patient type result in non-negative payoff for the provider.

We derive the equilibrium solution following the standard approach (Laffont and Martimort 2001; Chapter 7, Bolton and Dewatripont 2005; Chapter 6). A similar approach is used in other papers that consider both adverse selection and moral hazard (e.g., Yaesoubi et al. 2011, Xiao and Xu 2012, Chick et al. 2016). Specifically, first, based on the extended revelation principle (Laffont and Martimort 2001; page 258), we restrict our attention to direct mechanisms where the contracts are a pair of optimal treatment choices for the two patient types (i.e., $\left[\zeta_{L}, \tau_{L}, x_{L}\right]$ and $\left.\left[\zeta_{H}, \tau_{H}, x_{H}\right]\right)$. Next, because the provider is exposed to higher risk when treating high-risk patients, the provider's participation constraints require the contract for high-risk patients to have higher expected value than the contact for low-risk patients. Therefore, for highrisk patients the provider would not deviate to the contract designed for low-risk patients, and thus ICH will not bind at the optimum. Furthermore, the provider has an incentive to misrepresent low-risk patients as high-risk patients, so for low-risk patients the provider can 
benefit from his private information, and thus $I R L$ will not bind at the optimum. Thus, omitting $I C H$ and $I R L$, the constraints $I R H$ and $I C L$ are binding at the optimum for all treatment choices of the provider. ${ }^{1}$ Therefore, the payer's problem reduces to a maximization problem where the payer chooses optimal contracts subject to the two binding constraints (ICL and IRH). Since the provider's treatment threshold decision is private information (moral hazard), the payer chooses payment terms such that the provider's best response results in payer's optimal treatment threshold.

The underlying problem is novel in the sense that the provider has two decisions and the treatment threshold decision is conditional on the test-ordering decision. Moreover, the treatment threshold is the provider's only hidden action (moral hazard) whereas the test-ordering decision is public information. Therefore, if the provider does not order the test for a patient, the payer knows that the provider has chosen to treat the patient with advanced or basic treatment without knowing patient's recurrence probability (Option 0 or 1). Given that the treatment threshold decision is conditional on the test-ordering decision, the provider has nine treatment choices because there are two patient types and three treatment options $\left\{x_{i}, \tau_{i}\right\}$ per patient type. We solve the payer's maximization problem for each of the nine treatment choices (cf. Table A1 in the Appendix). In the equilibrium solution, given the model parameters the payer offers a menu of contracts that results in one of the nine treatment choices with the highest social welfare. The full details on the derivation of the equilibrium solution are shown in the Appendix.

\footnotetext{
${ }^{1}$ To relate to the literature in the supply chain coordination (e.g., Çakanyıldırım et al., 2012; Huang et al 2015; Yan et al., 2015), the provider with a high-risk patient corresponds to the supplier with a lower capability (high cost or low quality), and vice versa. Therefore, IRL and $I C H$ are binding in those papers, while $I R H$ and ICL are binding in our paper.
} 


\section{System Optimum}

In this section, we present the optimal solution for the system with information asymmetry (second-best policy). In section 4.1, we analyze a system with full information (first-best policy) to understand the inefficiencies caused by private information of the provider. We also explain how the proposed payment model outperforms current payment models in section 4.2.

Define $\hat{\tau}=\frac{z+c}{(1+\alpha) h+(1-k)\left(\lambda_{s}-\lambda_{r}\right)}$ as the critical treatment threshold; $\hat{\tau}$ is large when the advanced treatment's adverse events $(z)$ or cost $(c)$ is large, or when the cost associated with the provider's reputation concerns and altruistic behaviour $(\alpha)$ is small.

Lemma 1 presents a property of the optimal treatment threshold choice of the provider.

Lemma 1: When the provider orders the test $\left(x_{i}^{*}=1\right)$, the optimal treatment threshold is the same for both patient types and is equal to the critical treatment threshold $\left(i . e ., \tau_{i}^{*}=\hat{\tau}\right)$.

Lemma 1 states that in the optimal solution the payer designs contracts such that when the provider orders the test he will choose the same treatment threshold for both patient types that is equal to the critical treatment threshold. This is because the treatment threshold corresponds to the "marginal patient" for whom the provider is indifferent about treatment and the determination of this marginal patient is independent of the full distribution of risks for the patient type $F_{i}(r)$. Thus, the change to social welfare from treating or not treating a marginal patient with advanced treatment is independent of patients' risk distribution.

For expositional convenience, we define $\Gamma(\tau)=\alpha h \frac{\int_{0}^{\tau} F_{L}(y) d y-\int_{0}^{\tau} F_{H}(y) d y}{1-\tau+\int_{0}^{\tau} F_{H}(y) d y}$ where $\Gamma(\tau)$ can be interpreted as the provider's relative cost of not using advanced treatment for high-risk patients versus low-risk patients. The value $\Gamma(\tau)$ is zero when the distributions of risk of the two 
types are identical; it increases in the difference between the two types of patients and in the treatment threshold $\tau$. We define the following thresholds:

$$
\begin{gathered}
\bar{B}_{L}=\frac{z+c}{\hat{\tau}}\left\{\int_{0}^{\hat{\tau}} F_{L}(y) d y+\min \left[0, E\left[r_{L}\right]-\hat{\tau}\right]\right\} \\
\bar{B}_{H}=\frac{z+c}{\hat{\tau}}\left\{\int_{0}^{\hat{\tau}} F_{H}(y) d y-\frac{\beta}{1-\beta} \frac{\hat{\tau}}{z+c} \Gamma(\hat{\tau})+\min \left[0, E\left[r_{H}\right]-\hat{\tau}+\frac{\beta}{1-\beta} \frac{\hat{\tau}}{z+c} \Gamma(1)\right]\right\} \\
\bar{\tau} \text { solves } \hat{\tau}-\left(\frac{\beta}{1-\beta} \frac{\hat{\tau}}{z+c} \Gamma(\hat{\tau})+\int_{0}^{\hat{\tau}} F_{L}(y) d y-\int_{0}^{\hat{\tau}} F_{H}(y) d y\right)=E\left[r_{L}\right] \\
\hat{\tau}_{L}=E\left[r_{L}\right] \\
\hat{\tau}_{H}=\frac{E\left[r_{H}\right]}{1-\frac{\beta}{1-\beta} \frac{\Gamma(1)}{z+c}}
\end{gathered}
$$

Threshold $\bar{B}_{i}$ can be interpreted as the maximum willingness-to-pay for the test. There is a trade-off between the cost of the test and the expected risk to the patients. If the expected risk to the patients is much lower (higher) than the treatment threshold, then there is only a small probability that a patient would (would not) require advanced treatment given the test results. Therefore, as the distance between the expected risk and the treatment threshold $\hat{\tau}$ gets larger, the value of the test diminishes because the probability of making similar treatment decisions with and without the test gets higher.

Lemma 2: Define $\bar{z}=(1+\alpha) h+(1-k)\left(\lambda_{s}-\lambda_{r}\right)$ and $\bar{c}=(1+\alpha) h+(1-k)\left(\lambda_{s}-\lambda_{r}\right)-z$. If $z>\bar{z}$ or $c>\bar{c}$, then the patients are never treated with advanced treatment.

Lemma 2 shows that if the cost of adverse events is sufficiently large $(z>\bar{z})$ or the advanced treatment is sufficiently costly $(c>\bar{c})$, then the advanced treatment is never used. In 
that case, it is clearly never optimal to order the test. For the remainder of the paper, we focus on the interesting region where $z<\bar{z}$ and $c<\bar{c}$.

Proposition 1 presents the optimal solution for the system with information asymmetry (second-best policy), and is illustrated in Figure 3.

Proposition 1: The optimal solution for the system with information asymmetry is one of the following five cases:

- Case 1: For both patient types, do not order the test and treat the patients with advanced treatment if and only if $B>\bar{B}_{L}$ and $\hat{\tau}<\hat{\tau}_{L}$

- Case 2: For type-L patients, order the test and use advanced treatment for patients with $r>\hat{\tau} ;$ For type-H patients, do not order the test and treat the patients with advanced treatment if and only if $B \leq \bar{B}_{L}$ and $\hat{\tau}<\bar{\tau}$

- Case 3: For type-L patients, do not order the test and do not use advanced treatment; For type-H patients, do not order the test and treat the patients with advanced treatment if and only if $B>\max \left\{\bar{B}_{L}, \bar{B}_{H}\right\}$ and $\hat{\tau} \geq \hat{\tau}_{L}$ and $\hat{\tau}<\hat{\tau}_{H}$

- Case 4: For type-L patients, do not order the test and do not use advanced treatment; For type-H patients, order the test and use advanced treatment for patients with $r>\hat{\tau}$ if and only if $B \leq \bar{B}_{H}$ and $\hat{\tau} \geq \bar{\tau}$

- Case 5: For both patient types, do not order the test and do not use advanced treatment if and only if $B>\bar{B}_{H}$ and $\hat{\tau} \geq \hat{\tau}_{H}$

Consider the case where the cost of the diagnostic test is sufficiently high, such that the payer should not motivate the provider to order the test, regardless of the patient type (Cases 1, 3, 5). In all three cases, the potential benefit of ordering the test-that is, directing advanced 
treatment to those patients that derive the greatest benefit-does not justify the testing cost. It is analogous to public health and health policy guidelines (e.g., for screening, vaccination, etc.) where the guideline varies in the population prevalence. For example, one analysis of HIV screening concluded that one-time screening in the general population might be cost-effective in areas where HIV prevalence exceeds 3.0 percent (Paltiel et al. 2005).

Figure 3: Optimal treatment decisions for the system with information asymmetry

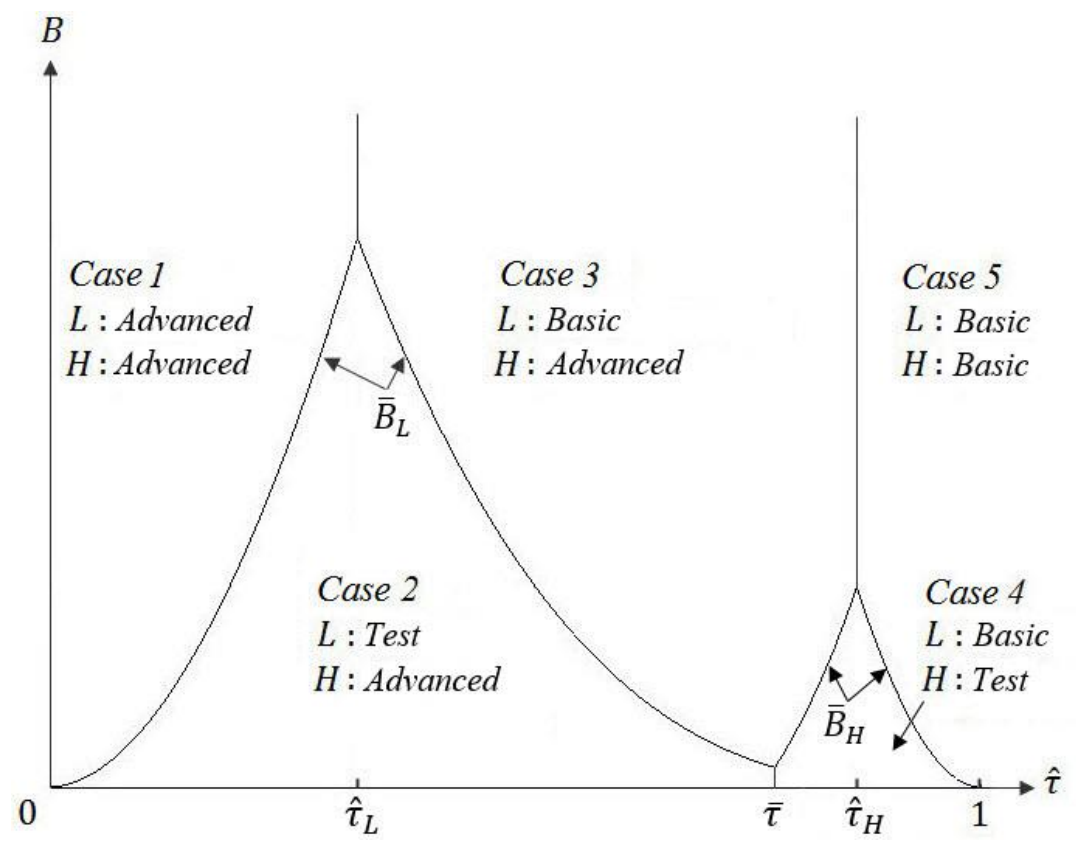

Next, consider the case where the diagnostic test is relatively inexpensive ( $B$ is small), such that social welfare may increase if the provider orders the test for the patients (Cases 2,4$)$. When the critical treatment threshold $\hat{\tau}$ is small (Case 2), the provider should test low-risk patients, and treat them with advanced treatment when their recurrence probability is larger than $\hat{\tau}$. However, in Case 2, high-risk patients are expected to have a recurrence risk larger than the threshold $\hat{\tau}$, so the payer should design a contract that motivates the provider to treat high-risk patients with advanced treatment without ordering the test. On the other hand, when the critical treatment threshold is large (Case 4), the provider should test high-risk patients and treat them 
with advanced treatment when their recurrence probability is larger than $\hat{\tau}$. However, in Case 4, low-risk patients are expected to have a recurrence risk lower than the critical treatment threshold, so the payer should design a contract that motivates the provider to treat low-risk patients with basic treatment without ordering the test.

Corollary 1 shows how the provider may benefit from his private information.

Corollary 1: In Cases 1, 2, and 3, the patient type does not affect the provider's profit. In Cases 4 and 5, the provider has a higher profit from treating low-risk patients than from treating highrisk patients.

In Cases 1, 2, and 3, the provider always treats high-risk patients with advanced treatment. As a result, the payer can design a contract for the provider for high-risk patients that (1) does not require any reward payments (i.e., $b_{n H}=0$ and $b_{t H}=0$ ), and (2) sets the fixed per patient treatment bundled payment for an episode of advanced treatment to be the cost of advanced treatment (i.e., $w_{H}=c$ ), leading to the provider having zero profit. Note that this contract structure is independent of the patients' risk distribution because there is no reward payments, so the provider with low-risk patients would also have the same profit if he were to pick this contract (incentive-compatibility constraint - ICL). As a result, the payer can design a contract such that the provider would also have zero profit from low-risk patients. Thus, patient type does not affect the provider's profit for these cases.

In Cases 4 and 5, the provider should not always treat high-risk patients with advanced treatment, so the payer must design a contract that includes reward payments for not using advanced treatment for high-risk patients (i.e., $b_{n H}>0$ ). While treating high-risk patients will have no profit for the provider (IRH is binding), the provider would have a positive profit when 
picking this contract for low-risk patients. This is because not treating low-risk patients with advanced treatment has less risk for the provider. Therefore, to prevent the provider from misrepresenting low-risk patients, the payer has to incentivize the provider by offering a profit larger than that offered for high-risk patients (i.e., information rent). In particular, the provider would have zero profit from high-risk patients in both cases, but the provider would have profit $\Gamma(\hat{\tau})$ in Case 4, and $\Gamma(1)$ in Case 5 from low-risk patients. (Recall that $\Gamma(\tau)$ is the provider's relative cost of not using advanced treatment for a patient. The argument of $\Gamma$ is $\hat{\tau}$ in Case 4 because the provider does not use advanced treatment for high-risk patients with recurrence risk smaller than $\hat{\tau}$, and the argument of $\Gamma$ is 1 in Case 5 because the provider never treats high-risk patients with advanced treatment, which is equivalent to setting the treatment threshold to one.)

Corollary 2 formally defines test cost thresholds beyond which it is never optimal to use the test.

Corollary 2: In the equilibrium solution, there exists an upper bound for the test cost such that

- if $B>\left.\bar{B}_{H}\right|_{\hat{\tau}=\hat{\tau}_{H}}$ then test is never ordered for high-risk patient type, and

- if $B>\left.\bar{B}_{L}\right|_{\hat{\tau}=\hat{\tau}_{L}}$ then test is never ordered for either patient type, and

- $\left.\bar{B}_{L}\right|_{\hat{\tau}=\hat{\tau}_{L}}>\left.\bar{B}_{H}\right|_{\hat{\tau}=\hat{\tau}_{H}}$.

Corollary 2 shows that regardless of the risk level of the patients, there is a level for test $\operatorname{cost}\left(\left.\bar{B}_{L}\right|_{\hat{\tau}=\hat{\tau}_{L}}\right)$ beyond which it never makes sense to use the test. It is intuitive that a threshold for the test cost exists, since there is a trade-off between the cost of the test and the value of the test in terms of improved treatment decisions. However, it may seem counterintuitive that this threshold is higher for low-risk patients (i.e., $\left.\bar{B}_{L}\right|_{\hat{\tau}=\hat{\tau}_{L}}>\left.\bar{B}_{H}\right|_{\hat{\imath}=\hat{\tau}_{H}}$ ). This is because in Case 4, the 
payer incurs information rent when motivating the provider to test high-risk patients (cf. Corollary 1). Therefore, ordering the test for high-risk patients will increase social welfare only when the test cost is low. On the other hand, in Case 2, the information rent is zero, and thus it is optimal for the payer to motivate the provider to test low-risk patients even at higher test cost amounts.

Corollary 3 presents a property of the optimal contracts (Table A2 in the Appendix presents the optimal contracts for each of the five cases in the equilibrium solution).

Corollary 3: In the optimal contracts, the provider never imposes a penalty on the provider.

The payer can offer a contract with only non-negative payment terms as long as the provider receives reward payments for stable health among patients who are not treated with advanced treatment (i.e., $b_{n i} \geq 0$ ). This is because the provider incurs a cost related to his reputation and his altruistic behaviour when a recurrence happens to patients treated with basic treatment, so the provider has an incentive to overuse advanced treatment. The proposed contracts counteract this by compensating the provider for stable health of patients who have not received advanced treatment. Note that the payer cannot reach a higher social welfare by including other contract terms, such as penalty payments for recurrence among patients treated with advanced or basic treatment. This is because the proposed payment model already incorporates the health outcome of patients in the payment terms. Thus, this result highlights the necessity of incentivizing the provider based on his treatment choice (such as incentivizing for not prescribing an episode of chemotherapy treatment for breast cancer patients). Having nonnegative payments is a desirable feature of contract design in healthcare applications because negative payments might be impossible to implement in practice and could cause additional 
incentive problems. A similar issue has been noted in other papers on the topic (Yaesoubi and Roberts 2011, Chick et al. 2016).

Note that when the reward payments are zero (i.e., $b_{t i}=b_{n i}=0$ ), the resulting contracts are simple bundled payments (i.e., $w_{i}$ ) that only pay for the quantity of the treatment without any consideration of the quality of the treatment. These types of contracts are similar to the commonly used FFS payment models. We show that when it is optimal to use the diagnostic test (Cases 2 and 4) the optimal contracts cannot be of this form (Table A2 in the Appendix). Consequently, the advent of new diagnostic tests makes current reimbursement models that only reward the intensity of work sub-optimal and necessitates development of alternative reimbursement models such as performance-based bundled payment models.

Corollary 4: Under the payment scheme proposed in this section, in the equilibrium of a system with information asymmetry it is never optimal to test patients from both patient risk profiles, even when the test is free (i.e., $B=0$ ).

Corollary 4 is true for all optimal cases presented in Proposition 1. In Case 2, where $\hat{\tau}$ is relatively small (Figure 3), advanced treatment's cost and adverse events are relatively small compared to the provider's cost when recurrence happens to patients treated with basic treatment. In this case, the provider uses advanced treatment for high-risk patients without ordering the test; accordingly, as stated in Corollary 1, the provider will have zero profit from treating both patient types. If the provider were to test high-risk patients, the test results might suggest that a high-risk patient does not need to be treated with advanced treatment; therefore, the provider would expect to have a disutility in case of a recurrence. This will require having higher-valued contract payment terms for high-risk patients if the provider were to order the test 
for high-risk patients in Case 2. Following higher-valued contracts for high-risk patients, to keep the provider from misrepresenting low-risk patients, the payer needs to offer higher-valued contracts for low-risk patients as well. As a result, in order to avoid the additional costs generated through higher-valued contract terms, in Case 2 the payer should not motivate the provider to order the test for high-risk patients.

In Case 4, the cost for a recurrence in patients treated with basis treatment is relatively small, and the provider treats low-risk patients with basic treatment without ordering the test. If the provider were to test low-risk patients, there is a small probability that the test suggests a low-risk patient to be treated with advanced treatment. However, the savings to the cost related to treating low-risk patients with advanced treatment (as it might be suggested by the test) does not justify higher payment terms that is required to motivate the provider to test low-risk patients. Thus, in Case 4 the payer should not motivate the provider to test low-risk patients, even when the test is free.

\subsection{Full information}

To examine whether the finding of Corollary 4 is due to decentralized decision-making or information asymmetry, we consider a system where there is a perfect information flow between the payer and the provider (first-best policy). The derivation of equilibrium of this system is presented in the Appendix, and the equilibrium solution is illustrated in Figure 4. The optimal treatment threshold in a system with perfect information is equal to the critical treatment threshold $(\hat{\tau})$ because it does not depend on the patients' risk profile and thus is not affected by information asymmetry in the system. Unlike the system with information asymmetry (Proposition 1), under complete information there is a new optimal case (Case 6) where the payer should motivate the provider to order the test for both patient types. This occurs when the test 
cost is sufficiently low. This is because, in the full information scenario, social welfare is only affected by the health outcome of patients and not by private information of the provider. In the full information scenario, advanced treatment is used only for a patient that can benefit from it. However, when the provider has private information, not all patients who require advanced treatment will receive it and not all the patients treated with advanced treatment will benefit from it. This finding illustrates the importance of information flow in a healthcare system in order to reduce the inefficiencies caused by the information asymmetry.

Figure 4: Optimal treatment decisions for the system with full information

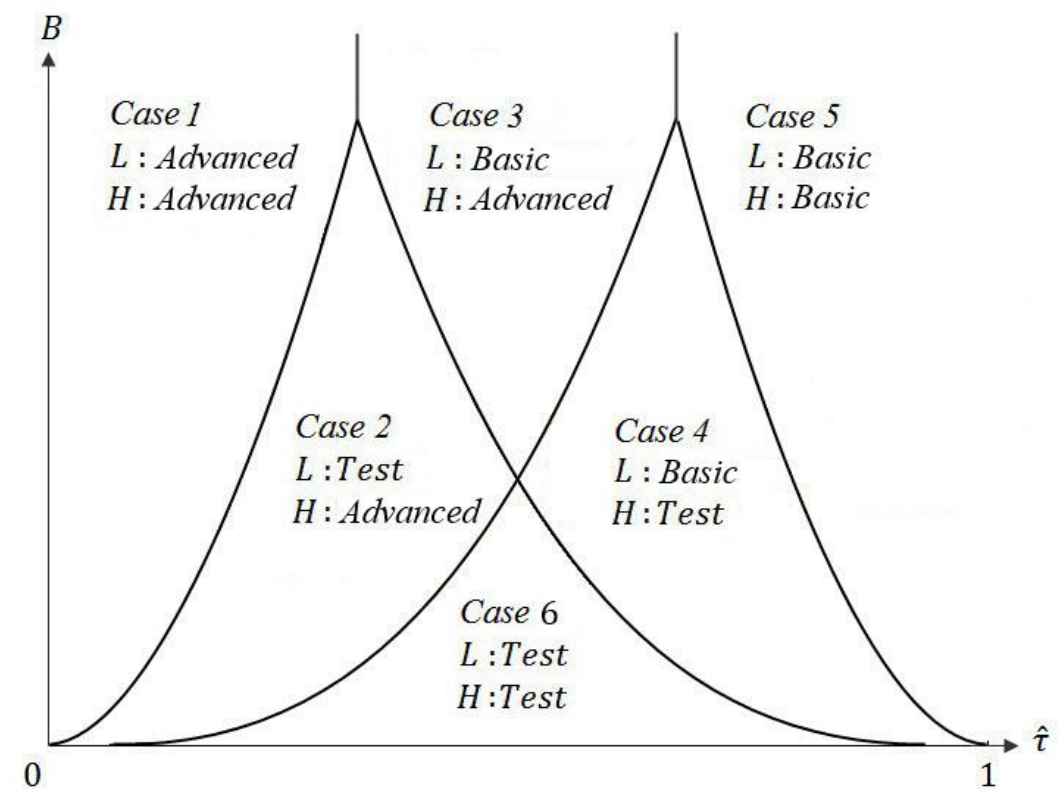

Remark 2: The highest willingness-to-pay for the test in the system with full information (firstbest policy) is independent of patients' risk profile, and is the same as the highest willingness-topay for the test in the system with information asymmetry $\left(\left.\bar{B}_{L}\right|_{\hat{\tau}=\hat{\tau}_{L}}\right)$. This is because in Case 2 the provider does not benefit from his private information and thus the payer's willingness-to-pay for the test is not affected by the information asymmetry. 


\subsection{Alternate payment models}

We also compare the proposed payment model with two other arrangements that are similar to currently implemented payment models. The derivation of equilibrium for these payment models are available from authors upon request.

First, we analyze the performance of a single performance-based payment contract that is offered to both patient types (i.e., $\zeta^{\prime}=\{w, b\}$ ) in a system with information asymmetry. This is similar to the OCM payment model. Under a single contract the payer cannot motivate the provider to treat each patient type differently. Therefore, the optimal treatment choices are Cases 1, 5 and 6. Note that, unlike in the full information scenario, in the presence of information asymmetry it is costly for the payer to test both patient types (Case 6) and thus this payment structure is always weakly dominated by our proposed payment model (i.e., $\zeta_{i}=\left\{w_{i}, b_{t i}, b_{n i}\right\}$ ).

Second, we analyze an alternative model where the payer offers a menu of contracts in which reward payments are only tied to the health outcome of a patient (i.e., $\zeta_{\boldsymbol{i}}^{\prime \prime}=\left\{w_{i}, b_{i}\right\}$ ). This is similar to the CJR payment model where a risk stratification methodology is used to set different target prices for different patient types. The optimal treatment choices under this model are qualitatively similar to the 5 cases presented in Figure 3 . However, the payment model that we propose can better utilize the optional diagnostic test by better managing the inefficiency in the system because by incorporating the treatment choice of the provider into the payment terms the payer can further reduce the information rent in Case 4. Both contract structures analyzed in this section have inferior performance to the proposed payment model, and hence emphasize the importance of development of alternative reimbursement models for different healthcare systems given the availability and use of personalized medicine. As in Fuloria and Zenios (2001) we investigated the implications of different payment models using an illustrative application. 
Figure 5 illustrates expected social welfare under each payment model using parameters listed in

Table 1 and full details are presented in Table A3 in the Appendix.

Table 1: Parameter estimations for numerical examples

\begin{tabular}{|l|l|l|}
\hline Model parameter & Estimated value & Source \\
\hline Cost for an episode of treatment $(c)$ & $\$ 30,000$ & Hornberger et al. $(2005) *$ \\
\hline Treatment adverse events $(z)$ & $\$ 19,000$ & Hornberger et al. $(2005)^{* *}$ \\
\hline Monetary benefit from stable health $\left(\lambda_{s}\right)$ & $\$ 100,000$ & Shiroiwa et al. $(2010) \dagger$ \\
\hline Monetary benefit from recurrence $\left(\lambda_{r}\right)$ & $\$ 60,000$ & Hornberger et al. $(2005) \ddagger$ \\
\hline Test cost $(B)$ & $\$ 4,175$ & Ray (2011) \\
\hline Hazard ratio $(k)$ & 0.95 & Paik et al. (2006) $\S$ \\
\hline Probability of type-L $\left(\beta_{L}\right)$ & 0.5 & Assumption \\
\hline Type-L patient distribution & $f_{L}(r)=2-2 r$ & Assumption II \\
\hline Type-H patient distribution & $f_{H}(r)=2 r$ & Assumption II \\
\hline
\end{tabular}

* Estimates of adjuvant chemotherapy costs vary depending on the type of regimen used. We estimate the cost of a treatment episode per course of trastuzumab.

**Average cost of minor, major, and fatal side effects.

$\uparrow$ Since health quality that is considered for reward payments is measured for a six-month period after an episode of treatment, we only consider willingness-to-pay for one additional quality-adjusted life-year (QALY) gained.

\$ Quality of life would drop to 0.7 when recurrence is stable, and to 0.5 when recurrence is progressive (base value 0.98). We use the average value of 0.6 to represent the average willingness-to-pay for QALY of patients with recurrence.

$\S$ Proportion of patients free of distant recurrence at 10 years for tamoxifen-treated patients and tamoxifen plus chemotherapy-treated patients across all risk groups.

II These distributions are assumed for illustrative purposes. We conducted experiments with other distributions and obtained qualitatively similar results.

Figure 5: Social welfare under different payment models

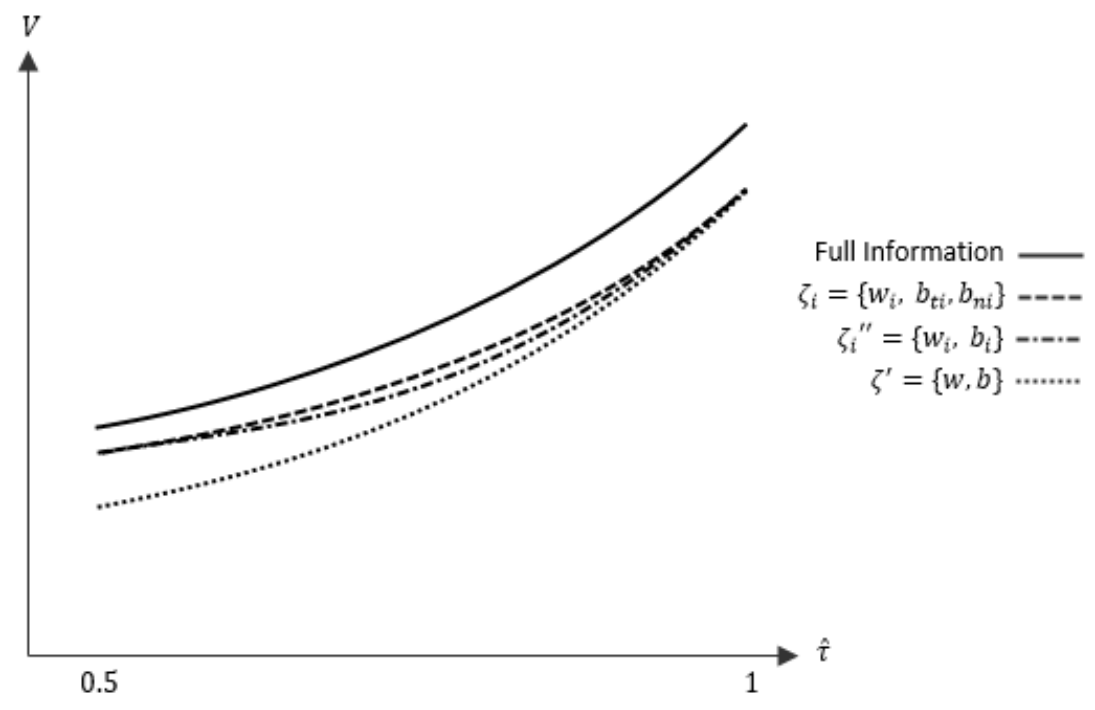




\section{Comparative Statistics}

In this section, we examine how social welfare is affected by treatment characteristics and the provider's concern for the health outcome of his patients.

For expositional convenience, we define:

$$
\Omega_{i}=1-\hat{\tau}+\int_{0}^{\hat{\tau}} F_{i}(y) d y
$$

Proposition 2 examines how social welfare is affected when patients treated with basic treatment experience a recurrence.

\section{Proposition 2:}

a) Social welfare increases when the welfare loss of a patient with recurrence who only received basic treatment has higher impact on the provider (i.e., $\alpha$ increases) if and only if

$$
B \leq \bar{B}_{H} \text { and } E\left[r_{L}\right] \leq \frac{\alpha h \hat{\tau}^{2}\left(F_{L}(\hat{\tau}) \Omega_{H}-F_{H}(\hat{\tau}) \Omega_{L}\right)-\left((z+c) \Omega_{H}-\alpha h \hat{\tau}^{2}\right)\left(\Omega_{L}-\Omega_{H}\right)}{(z+c) \Omega_{H}{ }^{2}}-\frac{(1-\beta)\left(\int_{0}^{\hat{\tau}} y f_{H}(y) d y\right)}{\beta} .
$$

b) Social welfare always decreases when the welfare loss of a patient with recurrence who only received basic treatment $(h)$ increases.

One might expect that, due to a more restrictive participation constraint and the resulting overtreatment of patients, social welfare cannot increase when the provider is more concerned about his reputation and his patients' health. However, Proposition 2.a formally states that this intuition does not always hold. In particular, when the provider does not treat low-risk patients with advanced treatment but uses the test for high-risk patients (Case 4), social welfare might increase as reputational concern and altruistic behaviour of the provider increases. This result arises because when $\alpha$ increases, the provider treating high-risk patients, in order to decrease his risk exposure, would use advanced treatment with higher probability (i.e., $\tau_{H}^{*}$ increases), which 
causes a decrease in the difference in the payments between low-risk and high-risk patient types. This allows the payer to design a contract such that the surplus utility for the provider treating low-risk patients to be smaller, resulting in an increase in the payer's objective function (maximizing social welfare). Note that, this is only true when the expected recurrence probability of low-risk patients is sufficiently small that it results in a higher surplus utility for the provider when treating low-risk patients. The higher the surplus utility of the provider, the higher the potential gain for social welfare. Another interpretation for this finding that the social welfare might increase as $\alpha$ increases could be that when $\alpha$ is large, the provider becomes completely altruistic or has sufficiently high reputational concerns that there is reduced need to incentivize the provider through the reimbursement mechanism. Proposition 2.a is illustrated in Figure 6 using parameters listed in Table 1.

Proposition 2.b shows that we cannot observe the same impact on social welfare when the welfare loss of a patient with recurrence who only received basic treatment $(h)$ increases. This is because an increase in the welfare loss of a patient has a direct negative impact on social welfare and cannot be compensated for by reducing the surplus utility of the provider.

Figure 6: Social welfare as a function of $\alpha$ multiplier in Case 4

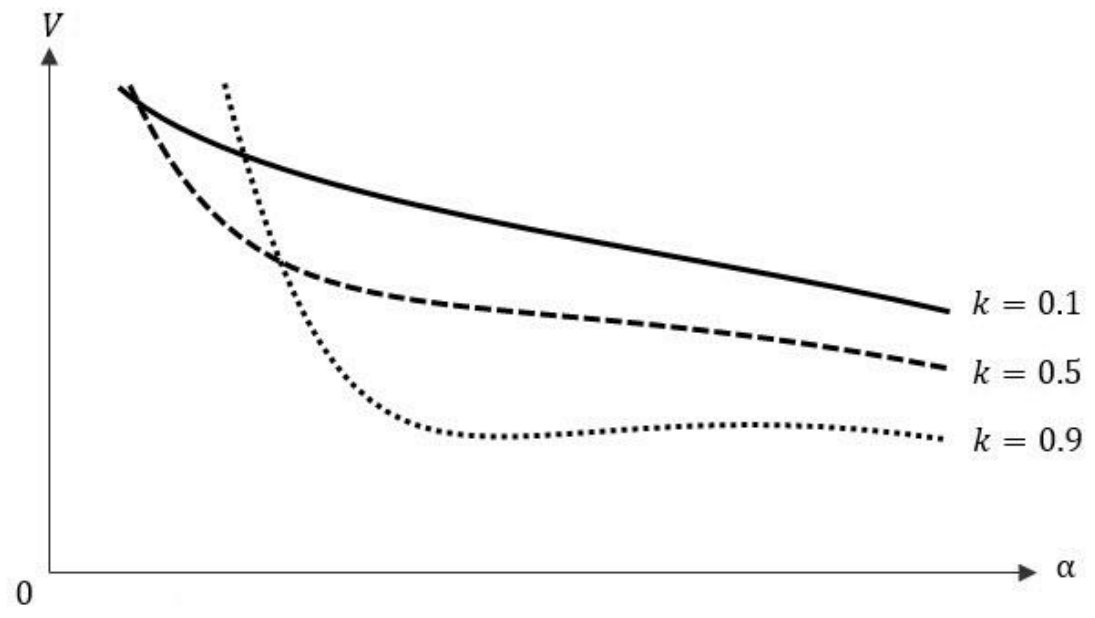


Lastly, the impact of changes in advanced treatment's adverse events $z$, cost $c$ and hazard ratio $k$ are intuitive and formally stated in Proposition 3.

Proposition 3: All else equal, the payer is always worse off as the advanced treatment becomes less desirable (i.e., increase in $z, c$, or $k$ ), and this effect of treatment characteristics on the social welfare is always larger when the treatment threshold is relatively small.

\section{Compulsory Testing}

There is a wide diversity in guidelines and recommendations for new diagnostic tests. In addition, many major guidelines are only updated every few years meaning that guidelines may lag technical developments. For instance, the 21-gene assay test for breast cancer is recommended by the American Society of Clinical Oncology and the U.S. National Comprehensive Cancer Network guidelines to be part of the routine practice for early stage breast cancer patients but not required as part of treatment (Ragaz 2010). Some other tests, such as "Tissue of Origin" test for cancer of unknown primary, are currently not recognized by any guidelines and the use of these tests depends on the physician's decision. Other tests are compulsory; for example, KRAS test must be performed before treatment with cetuximab or panitimuab for colorectal cancer in Ontario.

In this section, we assume that the payer might make the use of diagnostic tests compulsory and implement a mechanism to verify test results before reimbursing treatment. We assume that in making the test compulsory the payer incurs a verification $\operatorname{cost} C_{V}$ to gather and/or interpret the results - otherwise there would still be a problem with asymmetric information.

The payer's problem when the test is compulsory is as follows: 


$$
\begin{gathered}
\max _{\zeta} V^{\text {com }}=v(\zeta, 1, \tau)-C_{V} \\
\text { s.t } \tau^{*}=\underset{0 \leq \tau \leq 1}{\operatorname{argmax}} g(1, \tau) \\
g\left(1, \tau^{*}\right) \geq 0
\end{gathered}
$$

Program (12) is the modified version of the program (5) in the main model. The main difference is that there is no adverse selection because the payer acquires patient information and the test is used for all patients regardless of patients' risk profile. However, we assume that there is still moral hazard because the payer cannot impose a treatment choice, as recommended by the test result, to the provider. Since the test is compulsory the provider always orders the test (i.e., $x=1$ ). Lemma 3 presents the region in which the payer can improve social welfare by making the test compulsory.

Lemma 3: Define $\bar{B}^{\text {com }}=\frac{z+c}{\hat{\tau}}\left\{(1-\beta)\left(\int_{0}^{\hat{\tau}} F_{H}(y) d y+\min \left[0, E\left[r_{H}\right]-\hat{\tau}+\frac{\beta}{1-\beta} \frac{\hat{\tau}}{z+c} \Gamma(1)\right]\right)+\right.$ $\left.\beta\left(\int_{0}^{\hat{\tau}} F_{L}(y) d y+E\left[r_{L}\right]-\hat{\tau}\right)\right\}$. Then, $\left\{V^{\text {com }}>V \mid C_{V}=0\right\}$ if and only if:

- $\quad B \leq \frac{z+c}{\hat{\tau}} \int_{0}^{\hat{\tau}} F_{H}(y) d y$ and $\hat{\tau} \leq\left(\int_{0}^{\hat{\tau}} \mathrm{F}_{H}(y) d y+\int_{0}^{\hat{\tau}} \mathrm{F}_{L}(y) d y\right) /\left(2 \int_{0}^{\hat{\tau}} f_{L}(y) d y-1\right) ; O r$

- $\quad B \leq \bar{B}^{\text {com }}$ and $\hat{\tau}>\left(\int_{0}^{\hat{\tau}} \mathrm{F}_{H}(y) d y+\int_{0}^{\hat{\tau}} \mathrm{F}_{L}(y) d y\right) /\left(2 \int_{0}^{\hat{\tau}} f_{L}(y) d y-1\right)$

Proposition 4: It is not always socially optimal to make a diagnostic test compulsory even if such a policy can be implemented for free (i.e., $C_{V}=0$ ). 
Figure 7: Compulsory test versus Optional test when $C_{V}=0$

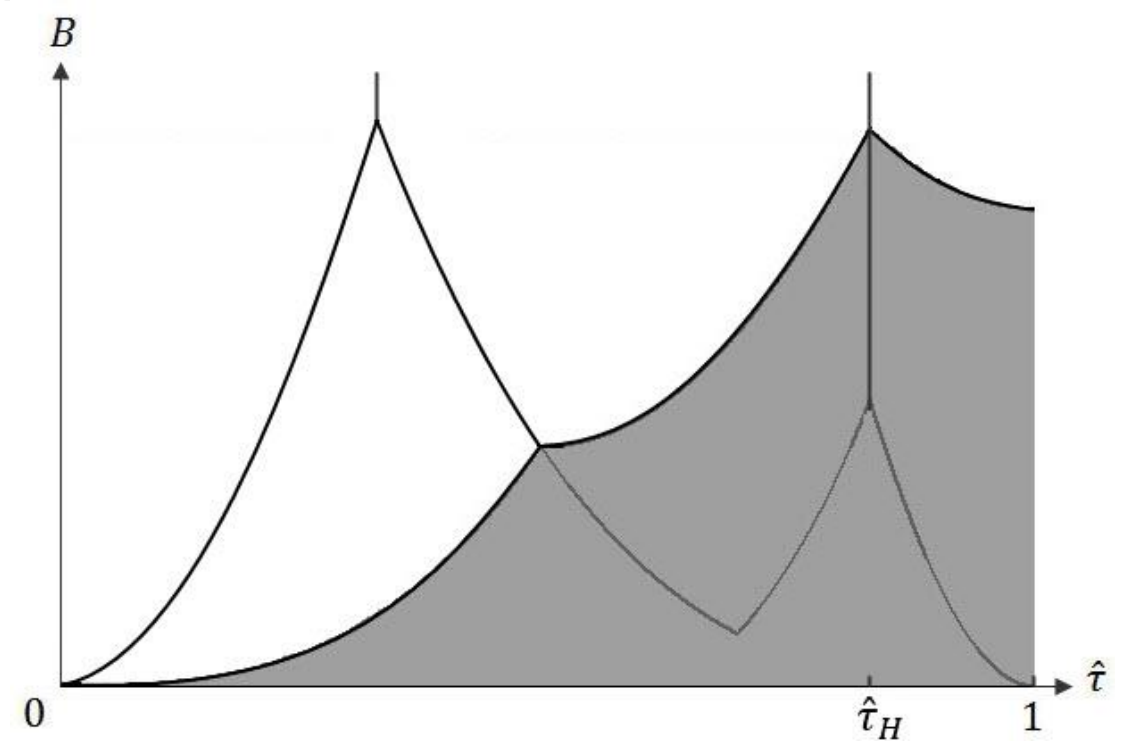

Proposition 4 is illustrated in Figure 7. The shaded area in Figure 7 is the region where social welfare is higher when the test is compulsory. To make it easier to compare with the original case where the test is optional, we have superimposed the shaded area on top of the graph from Figure 3 using the same scale on the y-axis. Figure 7 shows that while making the diagnostic test compulsory removes the adverse selection, even if the payer can implement this policy for free (i.e., $C_{V}=0$ ) it is not always optimal to do so. This is because the test result does not have much value for patients whose expected recurrence probability is higher than the optimal treatment threshold $(\hat{\tau})$. Thus when $\hat{\tau}$ is small, if the test is used for all patients, the payer pays for a test that might only change the treatment choice for a small group of patients. However, the compulsory testing policy becomes more effective when the optimal treatment threshold is relatively large, such that there is a higher probability for patients to have recurrence score below the treatment threshold and thus a diagnostic test is needed to eliminate unnecessary use of advanced treatment for low-risk patients. 
Corollary 5: There exists an upper bound for the test cost such that if $B>\left.\bar{B}^{\text {com }}\right|_{\hat{\tau}=\hat{\tau}_{H}}$ it is never optimal to make the test compulsory.

Corollary 5 formally shows the maximum test cost beyond which it is never optimal to make the test compulsory. However, as the verification cost for implementation of the policy increases, the shaded region in Figure 7 will shift down and compulsory testing will be optimal in a smaller region. Therefore, we can argue that while the maximum verification cost that justifies the compulsory testing depends on the test cost $(B)$, this threshold cannot be larger than the maximum test cost that makes the compulsory test optimal when $C_{V}=0$ (Lemma 3).

\section{Conclusions}

In this paper we examined the optimal design of contracts between a payer and a provider when an optional diagnostic test can be used to predict the benefits of an advanced treatment option. Following the general framework of the OCM, the contracts include reward payments based on the health of patients at the end of a review period. The provider has private information about patients' characteristics (adverse selection), and about whether or not the optimal course of action is used (moral hazard).

Our research provides an argument for the necessity of developing alternative payment models. Currently, performance-based payment models consider only the health outcome of a patient as the performance measure. However, we show that the payer can improve social welfare by incorporating the treatment choice of the provider in the payment contract. This payment structure can be easily adopted to the current performance-based payment models by including payments for stable health of patients that are not treated with an advanced treatment 
option. Our findings also highlight the potential impact of tort reform on social welfare, as well as highlighting the situations where a compulsory testing policy would be beneficial.

Similar to other studies in healthcare operations management (e.g., Jiang et al. 2012, Chick et al. 2016), we find an optimal menu of contracts assuming ex-ante hidden information. However, despite strong theoretical arguments favoring this type of contract, many contracts are simple and menus of contracts are rarely observed in practice (Gottlieb and Moreira 2015). One reason for this could be that creating incentives through a menu of contracts requires incentives based on the agent's priorities and goals, which may change over time (Bajari and Tadelis 2001). Thus, payers may look to methods other than optimal contract design to improve outcomes and reduce problems associated with adverse selection, such as more detail in their databases and enhanced information flow.

However, this paper is based on a stylized mathematical model with several limiting assumptions. While it is common to only study two types in systems with adverse selection (e.g., Yaesoubi and Roberts 2011, Çakanyıldırım et al. 2012, Xiao and Xu 2012, Saghafian and Chao 2014, Yan et al. 2015) the analysis for multiple types can be derived using similar methodology (see Bolton and Dewatripont 2005). In this case, there will be multiple perceived risk distributions for patients (i.e., $F_{n}(r)<F_{n-1}(r)<\cdots<F_{1}(r)$ where $F_{1}(r)$ is the distribution for the patients with the lowest risk and $F_{n}(r)$ is the distribution for the patients with the highest risk). The payer will offer a menu of contracts to the provider by considering the provider's incentive-compatibility and individual-rationality constraints. The provider will receive a zero profit from treating patients with highest risk $F_{n}(r)$, and the provider might have a positive surplus for all other patient types (information rent). The challenging part of the derivation is to reduce the incentive-compatibility constraints because the provider might have an incentive to 
misrepresent the patients from higher-risk distributions as patients from lower-risk distributions. However, given the structure of our findings, we expect that the main results of our paper (such as non-negative contract terms and not using the test for all patient types even if it was free) will continue to hold when there are multiple types. Generalization of the underlying problem to other testing technologies may require explicit modeling of the sensitivity and specificity of the tests. We assumed that there is only one provider with a fixed number of visiting patients. If patients have multiple options when deciding on which provider to visit, then a fruitful avenue of future research would be to consider competition among multiple providers. This scenario could be modelled as a dynamic principal-agent model, where the provider's reputation can affect the number of visiting patients in the future.

\section{Acknowledgments}

The authors are grateful to department editor Sergei Savin, special issue guest editor Kurt Bretthauer, the senior editor, and the two anonymous referees for their feedback. The authors are thankful to participants of the "Patient-Centric Healthcare Management in the Age of Analytics" conference held at Indiana University for their helpful comments. The

first author acknowledges support from the Ivey International Center for Health Innovation (IICHI). The second author acknowledges support from the Natural Sciences and Engineering Research Council of Canada (NSERC). 


\section{References}

Adida, E., H. Mamani, S. Nassiri. 2016. Bundled Payment vs. Fee-for-Service: Impact of Payment Scheme on Performance. Management Science 63(5) 1606-1624.

Allegra, C. J., J. M. Jessup, M. R. Somerfield, et al. 2009. American Society of Clinical Oncology provisional clinical opinion: testing for KRAS gene mutations in patients with metastatic colorectal carcinoma to predict response to anti-epidermal growth factor receptor monoclonal antibody therapy. Journal of clinical oncology 27(12) 2091-2096.

American cancer society. 2016. Cancer facts and figures. Available at:

http://www.cancer.org/acs/groups/content/@ research/documents/document/acspc047079.pdf [Accessed February 3, 2016].

Andritsos, D. A., C. S. Tang. 2015. Incentive programs for reducing readmissions when patient care is co-produced. Working paper, HEC Paris, Paris, France.

Avraham, R., M. Schanzenbach, 2010. The impact of tort reform on private health insurance coverage. American law and economics review 12(2) 319-355.

Bajari, P., S. Tadelis. 2001. Incentives versus transaction costs: A theory of procurement contracts. Rand Journal of Economics 32(3) 387-407.

Bangsberg, D. R., F. M. Hecht, E. D. Charlebois, et al. 2000. Adherence to protease inhibitors, HIV-1 viral load, and development of drug resistance in an indigent population. Aids 14(4) 357-366.

Behl, A. S., K. A. B. Goddard, T. J. Flottemesch, D. Veenstra, R. T. Meenan, J. S. Lin, M. V. Maciosek. 2012. Cost-effectiveness analysis of screening for KRAS and BRAF mutations in metastatic colorectal cancer. JNCI: Journal of the National Cancer Institute 104(23) $1785-1795$.

Bolton, P., M. Dewatripont. 2005. Contract Theory. MIT press.

Çakanyildirim, M., Q. Feng, X. Gan, S. P. Sethi. 2012. Contracting and coordination under asymmetric production cost information. Production and Operations Management 21(2) $345-360$.

Campbell, T. 2015. 5 Freakishly Expensive Cancer Drugs. Motley Fool. Available at: http://www.fool.com/investing/general/2015/03/28/5-freakishly-expensive-cancerdrugs.aspx [Accessed February 3, 2016].

Carlson, J. J., J. A. Roth. 2013. The impact of the Oncotype Dx breast cancer assay in clinical practice: A systematic review and meta-analysis. Breast cancer research and treatment 141(1) 13-22.

Center for disease control and prevention. 2013. Leading Causes of Death. Available at: http://www.cdc.gov/nchs/fastats/leading-causes-of-death.htm [Accessed January 20, 2016]. 
Chick, S. E., S. Hasija, J. Nasiry. 2016. Information elicitation and influenza vaccine production. Operations Research 65(1) 75-96.

Cms.gov. 2015. Oncology Care Model. Available at: http://innovation.cms.gov/initiatives/Oncology-Care/ [Accessed September 8, 2015].

Cotet, A. M. 2012. The impact of noneconomic damages cap on healthcare delivery in hospitals. American law and economics review 14(1) 192-234.

Dai, T., M. Akan, S. Tayur. 2016. Imaging Room and Beyond: The Underlying Economics Behind Physicians' Test-Ordering Behavior in Outpatient Services. Manufacturing \& Service Operations Management 19(1) 99-113.

Dai, T., X. Wang, C. W. Hwang. 2015. Clinical Ambiguity and Conflicts of Interest in Interventional Cardiology Decision-Making. Working paper, W. P. Carey School of Business, Baltimore, MD.

Davis, J., P. Ma, S. Saumya. 2010. The microeconomics of personalized medicine. McKinsey\&Company. Available at: http://www.mckinsey.com/industries/pharmaceuticals-and-medical-products/ourinsights/the-microeconomics-of-personalized-medicine [Accessed February 28, 2016].

Davis, S. 2014. Assessing Technologies That are Not Cost-effective at a Zero Price. Sheffield: NICE Decision Support Unit.

Davis, S., R. Akehurst. 2016. How Do We Evaluate Technologies That Are Not Cost Effective at Zero Price? Value \& Outcomes Spotlight 2(4) 1-8.

Díaz-Rubio, E., A. Gómez-España, B. Massutí, et al. 2012. Role of Kras Status in Patients with Metastatic Colorectal Cancer Receiving First-Line Chemotherapy plus Bevacizumab: A TTD Group Cooperative Study. PLoS One 7(10) e47345.

Enewold, L., A. M. Geiger, J. A. Zujewski, L. C. Harlan. 2015. Oncotype Dx assay and breast cancer in the United States: usage and concordance with chemotherapy. Breast cancer research and treatment 151(1) 149-156.

Evans, M. 2014. Incentive-based contracts thriving in commercial insurance market. Modern Healthcare. Available at: http://www.modernhealthcare.com/article/20140930/NEWS/309309931 [Accessed September 30, 2014].

Ferris, R., C. Blaum, E. Kiwak, J. Austin, J. Esterson, G. Harkless, G. Oftedahl, M. Parchman, P. Van Ness, M. E. Tinetti. 2017. Perspectives of patients, clinicians, and health system leaders on changes needed to improve the health care and outcomes of older adults with multiple chronic conditions. Journal of Aging and Health 1-22.

Fuloria, P. C., S. A. Zenios. 2001. Outcomes-Adjusted Reimbursement in a Health-Care Delivery System. Management Science 47(6) 735-751.

Gottlieb, D., H. Moreira. 2015. Simple contracts with adverse selection and moral hazard. 
Grundy, S., D. Becker, L. T. Clark, et al. (2002). Detection, evaluation, and treatment of high blood cholesterol in adults (Adult Treatment Panel III). Circulation-Hagertown 106(25) 3143.

Guo, P., C. S. Tang, Y. Wang, M. Zhao. 2016. The Impact of Reimbursement Policy on Patient Welfare, Readmission Rate and Waiting Time in a Public Healthcare System: Fee-forService vs. Bundled Payment. Working paper, the Hong Kong Polytechnic University, Hong Kong.

Gupta, D., M. Mehrotra. 2015. Bundled Payments for Healthcare Services: Proposer Selection and Information Sharing. Operations Research 63(4) 772-788.

Hannouf, M. B., E. Winquist, S. M. Mahmud, et al. 2016. Cost-effectiveness of using a gene expression profiling test to aid in identifying the primary tumour in patients with cancer of unknown primary. The pharmacogenomics journal 17(3) 286-300.

Hannouf, M. B., B. Xie, M. Brackstone, G. S. Zaric. 2012. Cost-effectiveness of a 21-gene recurrence score assay versus Canadian clinical practice in women with early-stage estrogen- or progesterone-receptor-positive, axillary lymph-node negative breast cancer. BMC Cancer 12(1) 447.

Hornberger, J., L. Cosler, G. Lyman. 2005. Economic analysis of targeting chemotherapy using a 21-gene RT-PCR assay in lymph-node-negative, estrogen-receptor-positive, early-stage breast cancer. The American Journal of Managed Care 11(5) 313-324.

Hornberger, J., R. Chien, K. Krebs, L. Hochheiser. 2011. US insurance program's experience with a multigene assay for early-stage breast cancer. Journal of oncology practice 7(3S) e38s-e45s.

Huang, H., X. Shen, H. Xu. 2015. Procurement Contracts in the Presence of Endogenous Disruption Risk. Decision Sciences 47(3) 437-472.

Japsen, B. 2015. Medicare's Bundled Payment Loses Some Interest Among Providers. Forbes. Available at: http://www.forbes.com/sites/brucejapsen/2015/12/15/medicares-bundledpayment-loses-some-interest-among-providers/\#7466e89011ce [Accessed September 18, 2016].

Jiang, H., Z. Pang, S. Savin. 2012. Performance-Based Contracts for Outpatient Medical Services. Manufacturing \& Service Operations Management 14(4) 654-669.

Jiang, H., Z. Pang, S. Savin. 2016. Improving Patient Access to Care: Performance Incentives and Competition in Healthcare Markets. Working paper, Wharton School, University of Pennsylvania, Philadelphia, PA.

Kaposy, C., N.R. Greenspan, Z. Marshall, J. Allison, S. Marshall, C. Kitson. 2017. Clinical ethics issues in HIV care in Canada: an institutional ethnographic study. BMC Medical Ethics 18(1) 9. 
Korda, H., G. N. Eldridge. 2011. Payment incentives and integrated care delivery: levers for health system reform and cost containment. The Journal of Health Care Organization, Provision, and Financing 48(4) 277-287.

Laffont, J. J., D. Martimort. 2001. The theory of incentives: the principal-agent model. Princeton university press.

Lakdawalla, D. N., S. A. Seabury. 2012. The welfare effects of medical malpractice liability. International review of law and economics 32(4) 356-369.

Montanera, D. 2016. The importance of negative defensive medicine in the effects of malpractice reform. The European Journal of Health Economics 17(3) 355-369.

Nerurkar, A., P. Osin, M. Parton. 2014. Integration of the 21-gene recurrence score assay results into the breast cancer assessment: An update for practicing surgical pathologists. The Journal of OncoPathology 2(1) 33-45.

Paik, S., G. Tang, S. Shak, et al. 2006. Gene expression and benefit of chemotherapy in women with node-negative, estrogen receptor-positive breast cancer. Journal of clinical oncology 24(23) 3726-3734.

Paltiel, A. D., M. C. Weinstein, A. D. Kimmel, et al. 2005. Expanded screening for HIV in the United States-an analysis of cost-effectiveness. New England Journal of Medicine 352(6) 586-595.

Picard, A. 2012. The price of life. The Globe and Mail. Available at: http://www.theglobeandmail.com/life/health-and-fitness/health/conditions/the-price-oflife/article623330/ [Accessed February 5, 2016].

Ragaz, J. 2010. The 21-gene assay: Canada's uneven response. Cancer Advocacy Coalition of Canada (cacc). Report Card on Cancer in Canada, 2011 41-3.

Ray, T. 2011. NanoString Study Suggests PAM50 Recurrence Test Provides Advantages over Oncotype DX. GenomeWeb. Available at: https://www.genomeweb.com/mdx/nanostringstudy-suggests-pam50-recurrence-test-provides-advantages-over-oncotyp [Accessed February 5, 2016].

Reyes, J. W. 2010. The effect of malpractice liability on the specialty of obstetrics and gynecology. Working paper (No. w15841), National Bureau of Economic Research.

Saghafian, S., X. Chao. 2014. The impact of operational decisions on the design of salesforce incentives. Naval Research Logistics 61(4) 320-340.

Savva, N., T. Tezcan, O. Yildiz. 2016. Yardstick Competition for Service Systems. Working paper, London Business School, London, UK.

Segurado, O. 2016. This is personal: Less chemotherapy for breast cancer. The Hill. Available at: http:/thehill.com/blogs/pundits-blog/healthcare/293822-this-is-personal-lesschemotherapy-for-breast-cancer [Accessed August 29, 2016]. 
Shiroiwa, T., Y. Sung, T. Fukuda. 2010. International survey on willingness-to-pay (WTP) for one additional QALY gained: what is the threshold of cost effectiveness? Health economics 19(4) 422-437.

Smith, T. J., B. E. Hillner. 2010. Concrete options and ideas for increasing value in oncology care: the view from one trench. Oncologist 15(Supplement 1) 65-72.

Tansey, B. 2008. Diagnostic test could pinpoint origins of cancers. SFGate. Available at: http://www.sfgate.com/business/article/Diagnostic-test-could-pinpoint-origins-ofcancers-3214832.php [Accessed February 5, 2016].

Vanderlaan, B. F., M. S. Broder, E. Y. Chang, R. Oratz, T. G. Bentley. 2011. Cost-effectiveness of 21-gene assay in node-positive, early-stage breast cancer. The American journal of managed care. 17(7) 455-464.

Whitman, E. 2016. Rapid adoption of bundled payments remains an act of faith. Modern Healthcare. Available at: http://www.modernhealthcare.com/article/20160922/NEWS/160929968?utm_source=mo dernhealthcare\&utm_campaign=am\&utm_medium=email\&utm_content=20160922NEWS-160929968 [Accessed September 23, 2016].

Xiao, W., Y. Xu. 2012. The Impact of Royalty Contract Revision in a Multistage Strategic R\&D Alliance. Management Science 58(12) 2251-2271.

Yaesoubi, R., S. D. Roberts. 2011. Payment contracts in a preventive healthcare system: A perspective from Operations Management. Journal of health economics 30(6) 11881196.

Yan, X., H. Zhao, K. Tang. 2015. Requirement or promise? An analysis of the first-mover advantage in quality contracting. Production and Operations Management 24(6) 917933. 